\section{Sequential endoscopic drainage and clip closure of an intrathoracic esophagogastric anastomotic dehiscence}
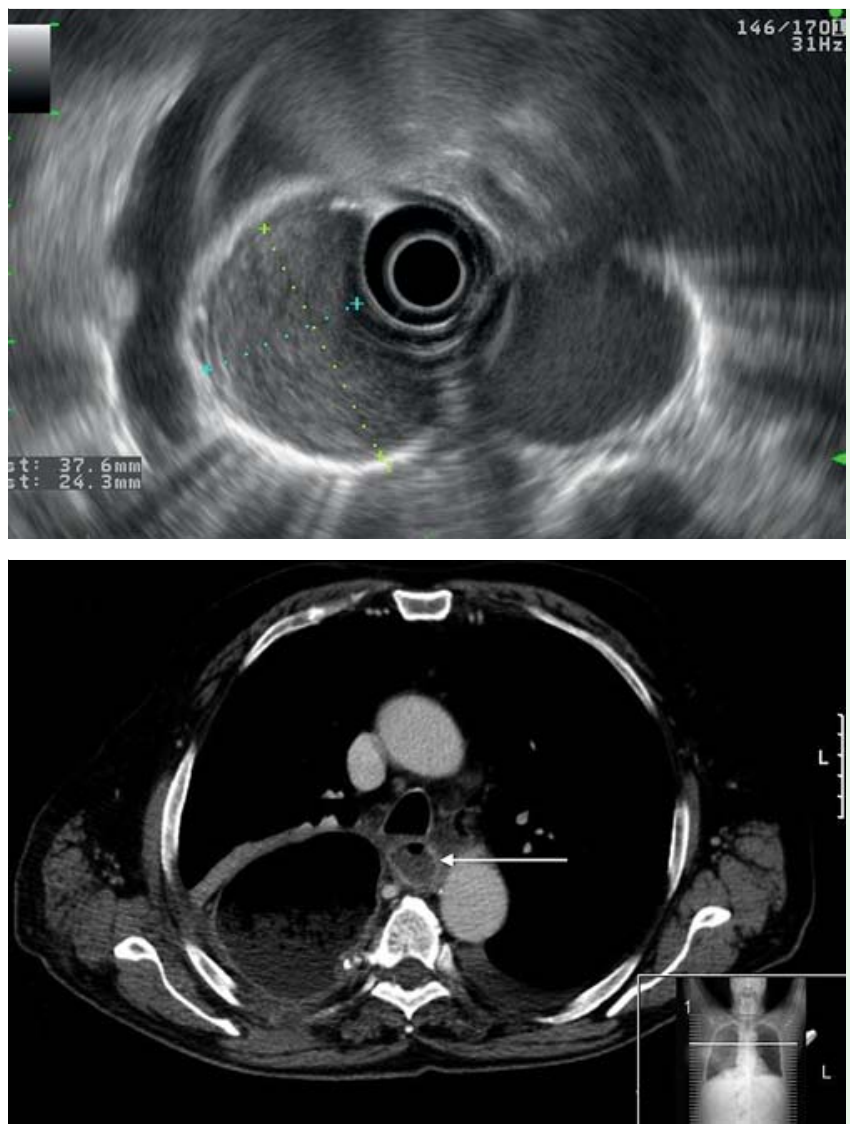

A 69-year-old man underwent an upper gastrointestinal endoscopy, which revealed a large submucosal lesion in the distal esophagus with normal overlying mucosa. Endoscopic ultrasound (EUS) showed a hypoechoic lesion measuring $80 \times 40 \times 30 \mathrm{~mm}$ in the submucosa ( $\bullet$ Fig. 1). A subtotal esophagectomy was performed. Histopathological examination of the resected tissue revealed a duplication cyst.

The patient developed sepsis 3 weeks after surgery. A computed tomography (CT) scan revealed evidence of dehiscence of the esophagogastric anastomosis with a large mediastinal collection ( $\bullet$ Fig. 2 ). Upper gastrointestinal endoscopy confirmed the presence of a dehiscence affecting an 8-mm section of the esophagogastric anastomosis ( $\mathbf{F i g . 3 a}$ ). Pus was aspirated through the fistula orifice and two double-pigtail plastic stents were placed endoscopically to drain the abscess
Fig. 2 Thoracic computed tomography (CT) scan showing a posterior mediastinal collection (arrow).

Fig. 1 Endoscopic ultrasound (EUS) view of the hypoechoic lesion in the submucosa of the distal esophagus.

( Fig. 3b). There was rapid improvement in the patient's clinical condition.

After 1 week, the stents were removed and an over-the-scope clip (OTSC) was applied to close the fistula. Unfortunately, despite this, a Gastrografin swallow revealed persistence of the fistula.

A third upper gastrointestinal endoscopy was performed and after removing the OTSC, we applied argon plasma coagulation (APC) and three through-the-scope clips, which effectively sealed the fistula ( $\bullet$ Fig.3c). Resolution of the mediastinal abscess as well as the fistula was confirmed on a further thoracic CT scan and Gastrografin swallow ( $\bullet$ Fig. 4).

This case highlights the potentially useful role of endoscopic drainage and clipping devices in the management of a rare but serious adverse event of esophageal surgery.

Endoscopy_UCTN_Code_TTT_1AO_2AI

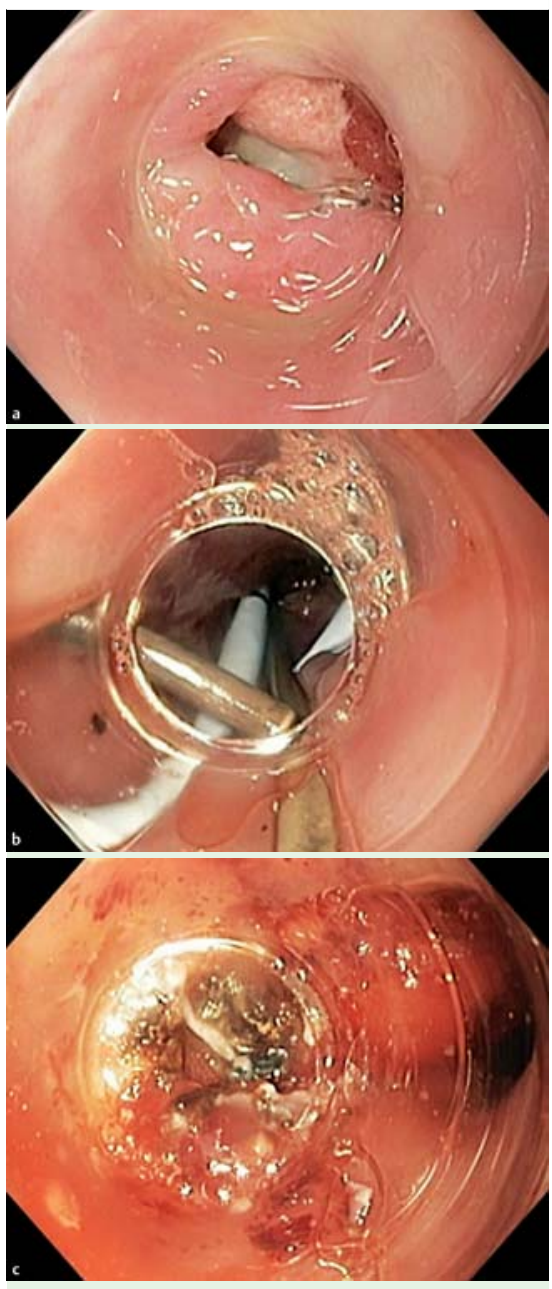

Fig. 3 Endoscopic views showing: a the esophagogastric anastomosis with an 8-mm long dehiscence; $\mathbf{b}$ two double-pigtail plastic stents passing through the fistula orifice to drain the mediastinal collection; $\mathbf{c}$ the application of argon plasma coagulation at the fistula orifice.

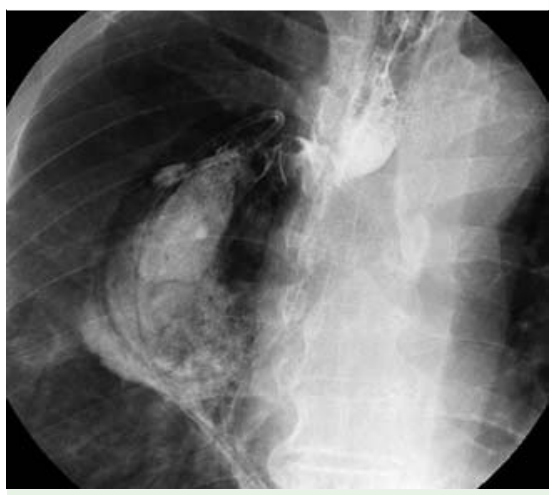

Fig.4 Gastrografin swallow after placement of a metal clip confirming that the fistula orifice had been successfully closed.

Competing interests: None 
Samuel Costa ${ }^{1}$, Carlos Ferreira', Rui Esteves ${ }^{2}$, Patrícia Lages ${ }^{2}$, Paulo Costa ${ }^{2}$, Luís Ribeiro ${ }^{1}$, José Velosa ${ }^{1}$

${ }^{1}$ Department of Gastroenterology and Hepatology, Hospital de Santa Maria, Lisbon, Portugal

2 Department of Surgery 1, Hospital de Santa Maria, Lisbon, Portugal
Bibliography

Dol http://dx.doi.org/

10.1055/s-0034-1377352

Endoscopy 2014; 46: E371-E372

(C) Georg Thieme Verlag KG

Stuttgart · New York

ISSN 0013-726X
Corresponding author

Samuel Costa, MD

Hospital de Santa Maria

Avenida Professor Egas Moniz

1649-035 Lisboa

Portugal

samuelcosta@hotmail.com 